\title{
A Hybrided Trapezoidal-Difference Scheme for Nonlinear Time-Fractional Fourth-Order Advection-Dispersion Equation Based on Chebyshev Spectral Collocation Method
}

\author{
Shichao $\mathrm{Yi}^{1,2}$ and Hongguang Sun ${ }^{2, *}$ \\ 1 School of Science, Jiangsu University of Science and Technology, Zhenjiang, Jiangsu \\ 212003, China \\ 2 Institute of Soft Matter Mechanics, College of Mechanics and Materials, Hohai \\ University, Nanjing, Jiangsu 210098, China
}

Received 3 February 2018; Accepted (in revised version) 16 August 2018

\begin{abstract}
In this paper, we firstly present a novel simple method based on a Picard integral type formulation for the nonlinear multi-dimensional variable coefficient fourthorder advection-dispersion equation with the time fractional derivative order $\alpha \in(1,2)$. A new unknown function $v(\mathbf{x}, t)=\partial u(\mathbf{x}, t) / \partial t$ is introduced and $u(\mathbf{x}, t)$ is recovered using the trapezoidal formula. As a result of the variable $v(\mathbf{x}, t)$ are introduced in each time step, the constraints of traditional plans considering the non-integer time situation of $u(\mathbf{x}, t)$ is no longer considered. The stability and solvability are proved with detailed proofs and the precise describe of error estimates is derived. Further, Chebyshev spectral collocation method supports accurate and efficient variable coefficient model with variable coefficients. Several numerical results are obtained and analyzed in multi-dimensional spatial domains and numerical convergence order are consistent with the theoretical value $3-\alpha$ order for different $\alpha$ under infinite norm.
\end{abstract}

AMS subject classifications: 65M60, 65N30, 65N15

Key words: Trapezoidal-difference scheme, time-fractional order, variable coefficient fourth-order advection-dispersion equation, Chebyshev spectral collocation method, nonlinearity.

\section{Introduction}

Fractional calculus is a natural generalization of integer order operator. Utilizing the models based on derivatives of fractional orders in several branches of science and engineering is a major study of many mathematicians and physicians [1-5]. Roughly speaking time fractional derivative is designed to characterize physical processes and dynamic systems with history memory. As a counterpart of traditional integer order differential

*Corresponding author.

Emails: shichaoyi@just.edu.cn (S. C. Yi), shg@hhu.edu.cn or sunhongguang08@gmail.com (H. G. Sun) 
equation, fractional differential equation can be obtained by replacing the integer order derivatives with fractional ones in integer order differential equation. Fractional partial differential equations(FPDEs), particularly space and time-fractional equations, have been widely studied to construct the existence of solution and validity of these problems [6-8]. In addition, the reliable and powerful numerical and analytical methods for solving FPDEs has been focused in the last two decades. According to the mathematical literature, fractional partial differential equations have been progressed in various problems in science and engineering such as the Schrödinger, diffusion and telegraph fractional equations [6,9-14].

In several applications, the fourth-order model system $[15,16]$ is an important part of the fractional order system and can be found in physics, engineering, statistics, and other fields, such as wave propagation in beam problems [17], A flat surface system of grooves [5,18], several mathematical models of fourth-order subdiffusion systems [18-21] and so on. Here we will consider the following the nonlinear multi-dimensional variable coefficient time-fractional fourth-order advection-dispersion equation:

$$
\begin{aligned}
& { }_{0}^{c} \mathfrak{D}_{t}^{\alpha} u(\mathbf{x}, t)-\left(A(\mathbf{x}, t)+{ }_{0}^{c} \mathfrak{D}_{t}^{\alpha}\right) \Delta u(\mathbf{x}, t) \\
= & -B(\mathbf{x}, t) \Delta^{2} u(\mathbf{x}, t)+\mathcal{N}(u(\mathbf{x}, t))+f(\mathbf{x}, t), \quad \mathbf{x} \in \Omega, \quad t \in(0, T],
\end{aligned}
$$

where $A(\mathbf{x}, t)$ and $B(\mathbf{x}, t)$ are positive variable coefficients with the following initial and boundary conditions:

$$
\begin{aligned}
& u(\mathbf{x}, 0)=u_{0}(\mathbf{x}), \quad u_{t}(\mathbf{x}, 0)=v_{0}(\mathbf{x}), \quad \mathbf{x} \in \Omega, \\
& u(\mathbf{x}, t)=\Delta u(\mathbf{x}, t)=0, \quad \quad \mathbf{x} \in \partial \Omega, \quad t \in(0, T],
\end{aligned}
$$

where $u(\mathbf{x}, t)$ is unknown functions. Here ${ }_{0}^{c} \mathfrak{D}_{t}^{\beta(\mathbf{x}, t)}$ denotes the higher order Caputo fractional derivative of variable order $\beta(\mathbf{x}, t)$ with respect to $t$ in $[4,5,11,15-21]$

$$
{ }_{0}^{c} \mathfrak{D}_{t}^{\beta(\mathbf{x}, t)} u(\mathbf{x}, t)=\frac{1}{\Gamma(n-\beta(\mathbf{x}, t))} \int_{0}^{t} \frac{\partial^{n} u(\mathbf{x}, \eta)}{\partial \eta^{n}} \frac{d \eta}{(t-\eta)^{\beta(\mathbf{x}, t)+1-n}}, \quad n-1 \leq \beta(\mathbf{x}, t) \leq n,
$$

where $\Gamma(\cdot)$ is the Gamma function. The nonlinear term $\mathcal{N}(u(\mathbf{x}, t))$ is assumed to satisfy the following conditions: a) $|\mathcal{N}(u(\mathbf{x}, t))| \leq C|u|$, b) The first-order derivative function of $\mathcal{N}(u(\mathbf{x}, t))$ with respect to $u$ is bounded, i.e., $\left|\mathcal{N}^{\prime}(u(\mathbf{x}, t))\right| \leq a, a$ is a positive constant.

Most of fractional partial differential equations do not have the analytic solutions, many researchers in the last two decades have focused on the approximation or numerical methods of these fractional order systems in [22,23]. Lots of the researchers focus their attention on the strong format. This format is directly obtained by the original discrete equation. So, it is also called the collocation method. Strong formulation is reliable, simple in structure, and easy to erect the algebra system. The homotopy analysis method was utilized to approximate some FPDEs in [24,25]. The finite difference scheme and fractional predictor-corrector method are introduced for simulating the multi-term timefractional wave-diffusion equations with computationally effective results by AdamsBashforth method [26]. Also, some fractional differential equations utilized for modeling dynamical systems are investigated by an implicit difference approximation in [27]. 
The Jacobi collocation method is used to find the numerical solution of the fractional advection-diffusion equation with a nonlinear source term by Parvizi et al. in [28] and so on.

Underlying model of time appropriate to the application of discrete schemes, almost these schemes need to consider the half time step situation of $u$ because it is easy to obtain the representations of the derivative situation and the integration process. Meanwhile the energy method of the $u$ commonly considers the variable value coupled with $v$. So, in this work the situation of $v$ is changed as the main consideration. The stability, solvability of the system are proved in detail and the convergence of $u$ is given with $3-\alpha$ order. Furthermore, the derivative operation is an unbounded operator, by constant, the integration is a refinement operator. In this paper, the integral formula coupled with the difference scheme is proved to be a good stable scheme in Section 4. Finally, the high temporal schemes, such as high order Lagrange scheme, high order Runge-Kutta method and so on, have high accuracy in time direction. However, it is regrettable that these schemes are hard to get the regularity in the time process. The conservation of energy is merely proved on these scheme and only on special conditions or semi-analytical method, such as, mass-conservative Fourier spectral methods [26] and so on.

Thus, we use the $v(t)$ to replace the the unknown value $u^{\prime}(t)$ in this paper. Let $\sigma(\mathbf{x}, t)=$ $\Delta u(\mathbf{x}, t)$ and this problem is rewritten as

$$
\begin{aligned}
& { }_{0}^{c} \mathfrak{D}_{t}^{\alpha}\left(u_{0}(\mathbf{x})+\int_{0}^{t} v^{\prime}(\mathbf{x}, \eta) d \eta\right)-\left(A(\mathbf{x}, t)+{ }_{0}^{c} \mathfrak{D}_{t}^{\alpha}\right) \Delta\left(u_{0}(\mathbf{x})+\int_{0}^{t} v^{\prime}(\mathbf{x}, \eta) d \eta\right) \\
& =-B(\mathbf{x}, t) \Delta \sigma+\mathcal{N}\left(u_{0}(\mathbf{x})+\int_{0}^{t} v^{\prime}(\mathbf{x}, \eta) d \eta\right)+f(\mathbf{x}, t), \\
& \sigma(\mathbf{x}, t)=\Delta\left(u_{0}(\mathbf{x})+\int_{0}^{t} v^{\prime}(\mathbf{x}, \eta) d \eta\right), \quad t \in(0, T],
\end{aligned}
$$

with the following initial and boundary conditions:

$$
\begin{array}{llrl}
v(\mathbf{x}, 0) & =v_{0}(\mathbf{x}), & & \mathbf{x} \in \Omega, \\
\sigma(\mathbf{x}, t)=v(\mathbf{x}, t)=0, & & \mathbf{x} \in \partial \Omega, \quad t \in(0, T] .
\end{array}
$$

On space discrete schemes, the Lagrange approach is a choice for dealing with polynomial interpolations in most cases. Taken as one of the high order lagrange schemes, Chebyshev collocation method is chosen as the priority scheme in this paper. Firstly, the Lemma 4.1 of this paper illustrates the hybrided trapezoidal-difference scheme based on Chebyshev collocation method keeping the regularity and conservation of energy in the time-difference process. Secondly, the Chebyshev collocation method has a good numerical stability and high accuracy. The key is that the Lagrange polynomial must be manipulated through the formulas of barycentric interpolation [29]. When the nodes are of uniform distribution, the weight functions becomes extremely big lead to the Runge phenomenon and ruins the merits of the lagrange interpolation. But, the family of Chebyshev points following the density proportion $\left(1-x^{2}\right)^{-1 / 2}$ has good numerical stability [30-35]. 
The collocation method based on Chebyshev polynomial interpolation was recently extended to solve various ordinary and partial differential equations including 1D high order initial and boundary values problems [36] and nonlinear Burgers' equation [37]. In fact, there are few reports about the application of barycentric lagrange interpolation in the literature, especially for the high dimensional problems.

In summary, the hybrided trapezoidal-difference scheme based on Chebyshev spectral collocation method is a stable, high accuracy scheme. The rest of the paper is organized as follows: In Section 2, the formula of a compact finite difference coupled with spectral collocation method is derived. In Section 3, some preliminary information and lemmas are presented. In Section 4, the stability, convergence and solvability are proofed. In Section 5, computational results for some numerical experiments are illustrated. Finally, some conclusions are discussed in Section 6.

\section{Formulation of time-fractional equation}

Let $\left\{t_{n} \mid n \geq 0\right\}$ is the uniform time interval, where $t_{n}=n \tau, \tau>0$. And the trapezoidaldifference scheme suppose

$$
u_{i}^{n}=u_{i}^{0}+\tau \sum_{k=1}^{n}\left(v_{i}^{k-1}+v_{i}^{k}\right) / 2, \quad \int_{0}^{t_{n}} g(\eta) \frac{\partial v_{i}}{\partial \eta} d \eta=\sum_{k=1}^{n}\left[\frac{v_{i}^{k}-v_{i}^{k-1}}{\tau} \int_{t_{k-1}}^{t_{k}} g(\eta) d \eta\right],
$$

where $u_{i}^{n}, v_{i}^{n}, A_{i}^{n}$ and $B_{i}^{n}$ are the values of function, the first derivative function, variable coefficients $A(\mathbf{x}, t)$ and $B(\mathbf{x}, t)$ at the time $t_{n}$ of the point $\mathbf{x}_{i}$, and $g(t)$ is the smooth function in $(0, T]$.

The difference scheme we will consider for (1.4) is as follows:

Case I: $n=1$

$$
\begin{aligned}
& \frac{1}{\tau \Gamma(2-\alpha)}\left[a_{0} v_{i}^{n}-\sum_{k=1}^{n-1}\left(a_{n-k-1}-a_{n-k}\right) v_{i}^{n}-a_{n-1} v_{i}^{0}\right] \\
& -\frac{1}{\tau \Gamma(2-\alpha)} \Delta\left[a_{0} v_{i}^{n}-\sum_{k=1}^{n-1}\left(a_{n-k-1}-a_{n-k}\right) v_{i}^{n}-a_{n-1} v_{i}^{0}\right] \\
= & \tau A_{i}^{n} \Delta\left(\sum_{k=1}^{n-1} v_{i}^{k}+\frac{v_{i}^{0}+v_{i}^{n}}{2}\right)-B_{i}^{n} \Delta \sigma_{i}^{n}+\mathcal{N}\left(u_{i}^{0}\right)+F_{i}^{n},
\end{aligned}
$$

Case II: $n \geq 2$

$$
\frac{1}{\tau \Gamma(2-\alpha)}\left[a_{0} v_{i}^{n}-\sum_{k=1}^{n-1}\left(a_{n-k-1}-a_{n-k}\right) v_{i}^{n}-a_{n-1} v_{i}^{0}\right]
$$




$$
\begin{aligned}
& -\frac{1}{\tau \Gamma(2-\alpha)} \Delta\left[a_{0} v_{i}^{n}-\sum_{k=1}^{n-1}\left(a_{n-k-1}-a_{n-k}\right) v_{i}^{n}-a_{n-1} v_{i}^{0}\right] \\
= & \tau A_{i}^{n} \Delta\left(\sum_{k=1}^{n-1} v_{i}^{k}+\frac{v_{i}^{0}+v_{i}^{n}}{2}\right)-B_{i}^{n} \Delta \sigma_{i}^{n}+2 \mathcal{N}\left(u_{i}^{n-1}\right)-\mathcal{N}\left(u_{i}^{n-2}\right)+F_{i}^{n},
\end{aligned}
$$

where

$$
a_{l}=\int_{t_{l}}^{t_{l+1}} \frac{d t}{t^{\alpha-1}}=\frac{1}{2-\alpha}\left[\left(t_{l+1}\right)^{2-\alpha}-\left(t_{l}\right)^{2-\alpha}\right]=\frac{\tau^{3-\alpha}}{2-\alpha}\left[(l+1)^{2-\alpha}-l^{2-\alpha}\right], \quad l \geq 0,
$$

and

$$
F_{i}^{n}=\Delta\left(u_{i}^{0}\right)-\Delta^{2}\left(u_{i}^{0}\right)+f_{i}^{n} .
$$

It is easy to verify that $\left\{a_{l}, 0 \leq l \leq n\right\}$ is a monotone decreasing sequence for each $n$ with $a_{0}=\tau^{2-\alpha} /(2-\alpha)$.

In the process of Chebyshev spectral collocation method, the essential part is the generation of the spectral differentiation matrix. We first give the Chebyshev spectral differentiation matrix on the interval $[-1,1]$. Other intervals can be easily handled by the pan and zoom.

The collocation points are chosen as Chebyshev-Gauss-Lobatto points $x_{k}=\cos ((k-$ $1) \pi /(m-1)), k=1,2, \cdots, m$. We assume that $u(x)$ is a smooth function on $[-1,1]$. Then $u(x)$ can be interpolated by a sum of smooth shape functions $\phi_{j}(x)$

$$
u_{m}(x)=\sum_{j=1}^{m} u\left(x_{j}\right) \phi_{j}(x)
$$

where

$$
\phi_{j}(x)=\left(\frac{\omega_{j}}{x-x_{j}} / \sum_{j=1}^{m} \frac{\omega_{k}}{x-x_{j}}\right) \quad \text { with } \omega_{j}^{-1}=\prod_{i=1, i \neq j}^{m}\left(x_{j}-x_{i}\right) .
$$

The derivatives of the interpolant $u_{m}(x)$ are then estimated at collocation points by differentiating (2.5)

$$
u_{m}^{\prime}(x)=\sum_{j=1}^{m} u\left(x_{j}\right) \phi_{j}^{\prime}(x)
$$

Define the vector of function and derivatives as

$$
U=\left[u\left(x_{1}\right), u\left(x_{2}\right), \cdots, u\left(x_{m}\right)\right]^{T}
$$

and

$$
U^{(1)}=\left[u^{\prime}\left(x_{1}\right), u^{\prime}\left(x_{2}\right), \cdots, u^{\prime}\left(x_{m}\right)\right]^{T},
$$

respectively. We can get the matrix formulation of (2.6) on collocation points

$$
U^{(1)}=D_{m} U,
$$


where $D_{m}$ is the first order Chebyshev spectral differentiation matrix. The entry of $D_{m}$ is

$$
\left(D_{m}\right)_{i j}= \begin{cases}\frac{\omega_{j}}{\omega_{i}} \frac{1}{x_{i}-x_{j}}, & \text { if } i \neq j, \\ \sum_{i=1, i \neq j}^{m}\left(\frac{\omega_{i}}{\omega_{j}} \frac{1}{x_{i}-x_{j}}\right), & \text { if } i=j .\end{cases}
$$

From the above analysis, we can also get the second order Chebyshev spectral differentiation matrix $D_{m}^{(2)}$ by differentiating the interpolant $u_{m}(x)$ twice. However, in this work, in order to construct a conservative scheme for the fourth order differential system, we compute the second order spectral differentiation matrix by $D_{m}^{(2)}=D_{m}^{2}$, the square of $D_{m}$. The matrix $D_{m}^{(2)}$ can map the vector $U$ to vector $U^{(2)}$ :

$$
U^{(2)}=D_{m}^{2} U,
$$

where $U^{(2)}=\left[u^{\prime \prime}\left(x_{1}\right), u^{\prime \prime}\left(x_{2}\right), \cdots, u^{\prime \prime}\left(x_{m}\right)\right]^{T}$ represents the second-derivative on collocation points. These spectral differentiation matrices have some useful properties.

The procedure given above for the calculation of the derivatives in one-dimension can be readily extended to two dimensions. If an unknown matrix $U$ is defined as $U\left(x_{i}, y_{k}\right)=u_{i k}$, then its partial derivatives evaluated at the collocation points can be expressed in terms of the matrix-matrix products, where the differentiation with respect to $x_{k}$ corresponds to multiplying the rows of $D_{x}$ (the collocation derivative matrix in the $x$-direction) by the columns of $U$, and the differentiation with respect to $y_{k}$ corresponds to multiplying the rows of $U$ by the columns of $D_{y}$ (the collocation derivative matrix transpose in the y-direction).

We also define

$$
\left\|g^{n}\right\|_{\infty}=\max _{1 \leq i \leq m}\left|g_{i}^{n}\right|, \quad\left|\nabla g^{n}\right|=\sqrt{D \sum_{i=1}^{m}\left(g_{i}^{n}\right)^{2}},
$$

where $D$ is the measurement of the space domain $\Omega$. In addition, if $g(\Gamma)=0$, we have

$$
\left\|g^{n}\right\|_{\infty} \leq \frac{\sqrt{D}}{2}\left|\nabla g^{n}\right|
$$

Then, we have the following error estimate from the interpolate process

Lemma 2.1 (see [38]). If $u \in H^{p}(\Omega)$ and $u_{\hat{m}} \in H^{\hat{m}}(\Omega)$, where $\Omega \in \mathbb{R}^{d}$ is a nonempty, open bounded set with a Lipschitz continuous boundary. Then the following error estimates for the lagrange interpolation collocation method holds

$$
\left\|\partial_{\mathbf{x}}^{l}\left(u-u_{\hat{m}}\right)\right\|_{l} \leq \hat{n}^{l-\min \{\hat{m}, p\}}\|u\|_{l}, \quad 0 \leq l \leq \min \{\hat{m}, p\},
$$

where $\hat{m}$ and $\hat{n}$ are the minimum polynomial order and the minimum number of nodes in spatial coordinates. 


\section{Preliminaries and some lemmas}

For the fractional derivation of the present scheme, we introduce the following lemmas from [39].

Lemma 3.1. For any $v=\left\{v\left(t_{0}\right), v\left(t_{1}\right), v\left(t_{2}\right), \cdots\right\}$, we have

$$
\begin{aligned}
& \sum_{n=1}^{m}\left[a_{0} v\left(t_{n}\right)-\sum_{k=1}^{n-1}\left(a_{n-k-1}-a_{n-k}\right) v\left(t_{k}\right)-a_{n-1} v\left(t_{0}\right)\right] v\left(t_{n}\right) \\
\geq & \frac{t_{m}^{1-\alpha}}{2} \tau \sum_{n=1}^{N} v\left(t_{n}\right)^{2}-\frac{t_{N}^{2-\alpha}}{2(2-\alpha)} v\left(t_{0}\right)^{2}
\end{aligned}
$$

where $a_{l}$ is defined in (2.4).

Lemma 3.2. For $n \geq 1$ and $t_{k}=k \tau, 0 \leq k \leq n$, we have

$$
\begin{aligned}
0 & \leq \sum_{k=1}^{n} \int_{t_{k-1}}^{t_{k}}\left[\left(t_{n}-t\right)^{2-\alpha}-\frac{\left(t-t_{k-1}\right)\left(t_{n}-t_{k}\right)^{2-\alpha}+\left(t_{k}-t\right)\left(t_{n}-t_{k-1}\right)^{2-\alpha}}{\tau}\right] d t \\
& \leq\left[\frac{2-\alpha}{12}+\frac{2^{3-\alpha}}{3-\alpha}-\left(1+2^{1-\alpha}\right)\right] \tau^{3-\alpha} .
\end{aligned}
$$

Lemma 3.3. Suppose $v(t) \in C^{2}\left(\left[0, t_{n}\right]\right)$. Then

$$
\begin{aligned}
& \left|\int_{0}^{t_{n}} v^{\prime}(t) \frac{d t}{\left(t_{n}-t\right)^{\alpha-1}}-\sum_{k=1}^{n} \frac{v\left(t_{k}\right)-v\left(t_{k-1}\right)}{\tau} \int_{t_{k-1}}^{t_{k}} \frac{d t}{\left(t_{n}-t\right)^{\alpha-1}}\right| \\
\leq & \frac{\tau^{3-\alpha}}{2-\alpha}\left[\frac{2-\alpha}{12}+\frac{2^{3-\alpha}}{3-\alpha}-\left(1+2^{1-\alpha}\right)\right]\|v(t)\|_{L^{2, \infty}\left(\left[0, t_{n}\right]\right)} .
\end{aligned}
$$

Lemma 3.4. Suppose $v(t) \in C^{2}\left[0, t_{n}\right]$. Then

$$
\begin{aligned}
& \left|\int_{0}^{t_{n}} v^{\prime}(t) \frac{d t}{\left(t_{n}-t\right)^{\alpha-1}}-\frac{1}{\tau}\left[a_{0} v\left(t_{n}\right)-\sum_{k=1}^{n-1}\left(a_{n-k-1}-a_{n-k}\right) v\left(t_{k}\right)-a_{n-1} v\left(t_{0}\right)\right]\right| \\
\leq & \frac{\tau^{3-\alpha}}{2-\alpha}\left[\frac{2-\alpha}{12}+\frac{2^{3-\alpha}}{3-\alpha}-\left(1+2^{1-\alpha}\right)\right]\|v(t)\|_{L^{1, \infty}\left(\left[0, t_{n}\right]\right)},
\end{aligned}
$$

where $a_{l}$ is defined in (2.4) and $1<\alpha<2$. 
Based on Lemmas 2.1 and 3.4, introducing Eqs. (2.2)-(2.3), we have

$$
\begin{aligned}
& { }^{c} \mathfrak{D}_{0}^{\alpha} u_{i}^{n}=\frac{1}{\Gamma(2-\alpha)} \int_{0}^{t_{n}} \frac{\partial^{2} u\left(\mathbf{x}_{i}, \tau\right)}{\partial \tau^{2}} \frac{d \tau}{\left(t_{n}-\tau\right)^{\alpha-1}} \\
& =\frac{1}{\tau \Gamma(2-\alpha)}\left[a_{0} v_{i}^{n}-\sum_{k=1}^{n-1}\left(a_{n-k-1}-a_{n-k}\right) v_{i}^{k}-a_{n-1} v_{i}^{0}\right]+c_{1}\left(\tau^{3-\alpha}+\hat{n}^{-\min \{\hat{m}, p\}}\right), \\
& { }^{c} \mathfrak{D}_{0}^{\alpha} \Delta u_{i}^{n}=\frac{1}{\Gamma(2-\alpha)} \int_{0}^{t} \frac{\partial^{2} \Delta u\left(\mathbf{x}_{i}, \tau\right)}{\partial \tau^{2}} \frac{d \tau}{\left(t_{n}-\tau\right)^{\alpha-1}} \\
& =\frac{1}{\tau \Gamma(2-\alpha)}\left[\Delta\left(a_{0} v_{i}^{n}-\sum_{k=1}^{n-1}\left(a_{n-k-1}-a_{n-k}\right) v_{i}^{k}-a_{n-1} v_{i}^{0}\right)\right]+c_{1}\left(\tau^{3-\alpha}+\hat{n}^{2-\min \{\hat{m}, p\}}\right),
\end{aligned}
$$

and

$$
A_{i}^{n} \Delta u_{i}^{n}-B_{i}^{n} \Delta^{2} u_{i}^{n}=\tau A_{i}^{n} \Delta\left(\sum_{k=1}^{n-1} v_{i}^{k}+\frac{v_{i}^{0}+v_{i}^{n}}{2}\right)-B_{i}^{n} \Delta \sigma_{i}^{n}+c_{2}\left(\tau^{3-\alpha}+\hat{n}^{4-\min \{\hat{m}, p\}}\right) .
$$

Considering the difference property of the nonlinear term $\mathcal{N}(u(\mathbf{x}, t))$,

$$
\mathcal{N}\left(u_{i}^{n}\right)=\left\{\begin{array}{ll}
\mathcal{N}\left(u\left(\mathbf{x}_{i}, t_{0}\right)\right)+c_{3}(\tau), & n=1 \\
2 \mathcal{N}\left(u\left(\mathbf{x}_{i}, t_{n-1}\right)\right)-\mathcal{N}\left(u\left(\mathbf{x}_{i}, t_{n-2}\right)\right)+c_{3}^{\prime}\left(\tau^{2}\right), & n \geq 2 .
\end{array}\right\} .
$$

Substituting above results into (1.1) and noticing the initial value condition, we obtain

$$
\begin{aligned}
& \frac{1}{\tau \Gamma(2-\alpha)}\left[a_{0} v_{i}^{n}-\sum_{k=1}^{n-1}\left(a_{n-k-1}-a_{n-k}\right) v_{i}^{n}-a_{n-1} v_{i}^{0}\right] \\
& +\frac{1}{\tau \Gamma(2-\alpha)}\left[\Delta\left(a_{0} v_{i}^{n}-\sum_{k=1}^{n-1}\left(a_{n-k-1}-a_{n-k}\right) v_{i}^{n}-a_{n-1} v_{i}^{0}\right)\right] \\
= & \tau A_{i}^{n} \Delta\left(\sum_{k=1}^{n-1} v_{i}^{k}+\frac{v_{i}^{0}+v_{i}^{n}}{2}\right)-B_{i}^{n} \Delta \sigma_{i}^{n}+\hat{F}_{i}^{n}+R_{i}^{n},
\end{aligned}
$$

where

$$
\left|R_{i}^{n}\right| \leq c\left(\tau^{3-\alpha}+\hat{n}^{4-\min \{\hat{m}, p\}}\right), \quad n>1,
$$

and $\hat{F}_{i}^{n}$ involves the original $F_{i}^{n}$ and the approximate value of $\mathcal{N}\left(u_{i}^{n}\right)$.

\section{Analysis of the hybrided trapezoidal-difference scheme}

Before we prove the solvability, stability and convergence, we first give the notation the inner product and the bilinear form by

$$
(u, w)=\int_{\Omega} u w d \Omega, \quad \forall u, w \in L^{2}(\Omega), \quad \text { especially, }(u, u)=\|u\|^{2}=\sum_{i=1}^{m} \mathfrak{w}_{i}\left(u_{i}\right)^{2},
$$


$\mathfrak{w}_{i}$ denotes the gauss weight at the corresponding gauss point $\mathbf{x}_{i}$. Then, we give some lemmas.

Lemma 4.1. Suppose $\left\{v^{n}\right\}$ is the solution of

$$
\begin{aligned}
& \frac{1}{\tau \Gamma(2-\alpha)}\left[a_{0} v_{i}^{n}-\sum_{k=1}^{n-1}\left(a_{n-k-1}-a_{n-k}\right) v_{i}^{n}-a_{n-1} v_{i}^{0}\right] \\
& +\frac{1}{\tau \Gamma(2-\alpha)}\left[\Delta\left(a_{0} v_{i}^{n}-\sum_{k=1}^{n-1}\left(a_{n-k-1}-a_{n-k}\right) v_{i}^{k}-a_{n-1} v_{i}^{0}\right)\right] \\
= & \tau A_{i}^{n} \Delta\left(\sum_{k=1}^{n-1} v_{i}^{k}+\frac{v_{i}^{0}+v_{i}^{n}}{2}\right)-B_{i}^{n} \Delta \sigma_{i}^{n}+R_{i}^{n}, \quad u_{i}^{n}(\partial \Omega)=0, \quad i=1,2, \cdots, \quad m, n=1,2, \cdots .
\end{aligned}
$$

We have

$$
\sum_{n=1}^{N}\left\|\nabla u^{n}\right\|^{2} \leq \sum_{n=1}^{N}\left\|\nabla u^{0}\right\|^{2}+\frac{t_{N}^{2-\alpha}}{4 \hat{C} \Gamma(3-\alpha)}\left(\left\|v^{0}\right\|^{2}+\left\|\nabla v^{0}\right\|^{2}\right)+\frac{\Gamma(2-\alpha) t_{N}^{\alpha-1}}{4 \hat{C}} \tau \sum_{n=1}^{N}\left\|R^{n}\right\|^{2}
$$

with $A_{i}^{n} \geq \hat{A}>0, B_{i}^{n} \geq \hat{B}>0$ and $\hat{C}=\min \{\hat{A}, \hat{B}\}$.

Proof. Multiplying both sides of (4.2) by $\mathfrak{w}_{i} v_{i}^{n}$ and summing up for $i$ from 1 to $m$ and for $n$ from 1 to $N$, we obtain

$$
\begin{aligned}
& \frac{1}{\tau \Gamma(2-\alpha)} \sum_{i=1}^{m}\left\{\sum _ { n = 1 } ^ { N } \mathfrak { w } _ { i } \left(\left[a_{0} v_{i}^{n}-\sum_{k=1}^{n-1}\left(a_{n-k-1}-a_{n-k}\right) v_{i}^{k}-a_{n-1} v_{i}^{0}\right]\right.\right. \\
& \left.\left.-\Delta\left[a_{0} v_{i}^{n}-\sum_{k=1}^{n-1}\left(a_{n-k-1}-a_{n-k}\right) v_{i}^{k}-a_{n-1} v_{i}^{0}\right]\right) v_{i}^{n}\right\} \\
= & \sum_{n=1}^{N} \sum_{i=1}^{m}\left(A_{i}^{n} \mathfrak{w}_{i}\left[\sum_{x \in \mathbf{x}} \delta_{x}^{2}\left(\sum_{k=1}^{n-1} v^{k}+\frac{v^{0}+v^{n}}{2}\right)\right] v_{i}^{n}\right) \\
& -\sum_{n=1}^{N} \sum_{i=1}^{m}\left(B_{i}^{n} \mathfrak{w}_{i}\left[\sum_{x \in \mathbf{x}} \delta_{x}^{2}\left(\sum_{k=1}^{n-1} \Delta v^{k}+\frac{\Delta v^{0}+\Delta v^{n}}{2}\right)\right] v_{i}^{n}\right)+\sum_{n=1}^{N} \sum_{i=1}^{m} \mathfrak{w}_{i} R_{i}^{n} v_{i}^{n} .
\end{aligned}
$$

Using Lemma 3.1, we have

$$
\begin{aligned}
& \frac{1}{\tau \Gamma(2-\alpha)} \sum_{n=1}^{N} \sum_{i=1}^{m}\left(\mathfrak{w}_{i}\left[a_{0} v_{i}^{n}-\sum_{k=1}^{n-1}\left(a_{n-k-1}-a_{n-k}\right) v_{i}^{k}-a_{n-1} v_{i}^{0}\right] v_{i}^{n}\right) \\
\geq & \frac{1}{2 \Gamma(2-\alpha)} t_{N}^{1-\alpha} \sum_{n=1}^{N}\left\|v^{n}\right\|^{2}-\frac{t_{N}^{2-\alpha}}{2 \tau \Gamma(3-\alpha)}\left\|v^{0}\right\|^{2},
\end{aligned}
$$


and

$$
\begin{aligned}
& \frac{1}{\tau \Gamma(2-\alpha)} \sum_{n=1}^{N} \sum_{i=1}^{m}\left(\mathfrak{w}_{i} \Delta\left[a_{0} v_{i}^{n}-\sum_{k=1}^{n-1}\left(a_{n-k-1}-a_{n-k}\right) v_{i}^{k}-a_{n-1} v_{i}^{0}\right] v_{i}^{n}\right) \\
\geq & \frac{1}{2 \Gamma(2-\alpha)} t_{N}^{1-\alpha} \sum_{n=1}^{N}\left\|\nabla v^{n}\right\|^{2}-\frac{t_{N}^{2-\alpha}}{2 \tau \Gamma(3-\alpha)}\left\|\nabla v^{0}\right\|^{2} .
\end{aligned}
$$

Applying the boundary conditions in (4.2), we have $v_{i}^{n}(\partial \Omega)=\nabla v_{i}^{n}(\partial \Omega)=0$. Consequently,

$$
\begin{aligned}
& \tau \sum_{i=1}^{m}\left(\mathfrak{w}_{i} \Delta\left(\sum_{k=1}^{n-1} v_{i}^{k}+\frac{v_{i}^{0}+v_{i}^{n}}{2}\right) v_{i}^{n}\right)=-\tau \sum_{i=1}^{m}\left(\mathfrak{w}_{i} \Delta\left(\sum_{k=1}^{n-1} v_{i}^{k}+\frac{v_{i}^{0}+v_{i}^{n}}{2}\right) v_{i}^{n}\right)=-\frac{\tau}{2}\left\|\sum_{k=1}^{n} \nabla v^{k}\right\|^{2}, \\
& -\tau \sum_{i=1}^{m}\left(\mathfrak{w}_{i} \Delta^{2}\left(\sum_{k=1}^{n-1} v_{i}^{k}+\frac{v_{i}^{0}+v_{i}^{n}}{2}\right) v_{i}^{n}\right)=-\tau \sum_{i=1}^{m}\left(\mathfrak{w}_{i} \Delta^{2}\left(\sum_{k=1}^{n-1} v_{i}^{k}+\frac{v_{i}^{0}+v_{i}^{n}}{2}\right) v_{i}^{n}\right) \\
& =-\frac{\tau}{2}\left\|\sum_{k=1}^{n} \Delta v^{k}\right\|^{2} .
\end{aligned}
$$

In addition,

$$
\sum_{n=1}^{N} \sum_{i=1}^{m} \mathfrak{w}_{i} R_{i}^{n} v_{i}^{n} \leq \frac{1}{2} \frac{1}{\Gamma(2-\alpha)} t_{N}^{1-\alpha} \sum_{n=1}^{N}\left\|v^{n}\right\|^{2}+\frac{\Gamma(2-\alpha)}{2} t_{N}^{\alpha-1} \sum_{n=1}^{N}\left\|R^{n}\right\|^{2} .
$$

Substituting (4.5)-(4.8) into (4.4), we obtain

$$
\begin{aligned}
& \frac{1}{2 \Gamma(2-\alpha)} t_{N}^{1-\alpha} \sum_{n=1}^{N}\left\|v^{n}\right\|^{2}-\frac{t_{N}^{2-\alpha}}{2 \tau \Gamma(3-\alpha)} \sum_{n=1}^{N}\left\|v^{0}\right\|^{2}+\frac{1}{2 \Gamma(2-\alpha)} t_{N}^{1-\alpha} \sum_{n=1}^{N}\left\|\nabla v^{n}\right\|^{2} \\
& -\frac{t_{N}^{2-\alpha}}{2 \tau \Gamma(3-\alpha)} \sum_{n=1}^{N}\left\|\nabla v^{0}\right\|^{2} \\
\leq & -\frac{\tau \hat{A}}{2} \sum_{n=1}^{N}\left\|\sum_{k=1}^{n} \nabla v^{k}\right\|^{2}-\frac{\tau \hat{B}}{2} \sum_{n=1}^{N}\left\|\sum_{k=1}^{n} \Delta v^{k}\right\|^{2}+\frac{1}{2 \Gamma(2-\alpha)} t_{N}^{1-\alpha} \sum_{n=1}^{N}\left\|v^{n}\right\|^{2} \\
& +\frac{\Gamma(2-\alpha)}{2} t_{N}^{\alpha-1} \sum_{n=1}^{N}\left\|R^{n}\right\|^{2} .
\end{aligned}
$$

Then,

$$
\sum_{n=1}^{N}\left\|\sum_{k=1}^{n} \nabla v^{k}\right\|^{2} \leq \frac{t_{N}^{2-\alpha}}{\hat{C} \tau^{2} \Gamma(3-\alpha)}\left(\left\|v^{0}\right\|^{2}+\left\|\nabla v^{0}\right\|^{2}\right)+\frac{\Gamma(2-\alpha) t_{N}^{\alpha-1}}{\hat{C} \tau} \sum_{n=1}^{N}\left\|R^{n}\right\|^{2} .
$$

Combing the correction between $v$ and $u$ with (4.10), the following inequality is obtained

$$
\begin{aligned}
& \sum_{n=1}^{N}\left\|\nabla u^{n}\right\|^{2} \leq \sum_{n=1}^{N}\left\|\nabla u^{0}\right\|^{2}+\left(\frac{\tau}{2}\right)^{2} \sum_{n=1}^{N}\left\|\sum_{k=1}^{n} \nabla v^{k}\right\|^{2} \\
\leq & \sum_{n=1}^{N}\left\|\nabla u^{0}\right\|^{2}+\frac{t_{N}^{2-\alpha}}{4 \hat{C} \Gamma(3-\alpha)}\left(\left\|v^{0}\right\|^{2}+\left\|\nabla v^{0}\right\|^{2}\right)+\frac{\Gamma(2-\alpha) t_{N}^{\alpha-1}}{4 \hat{C}} \tau \sum_{n=1}^{N}\left\|R^{n}\right\|^{2} .
\end{aligned}
$$


Thus, we complete the proof.

Theorem 4.1. The difference scheme (2.2)-(2.3) is uniquely solvable.

Proof. Since (2.2)-(2.3) are a system of linear algebraic equations at each iterative process of different time level, it suffices to show that the corresponding homogeneous equations:

$$
\begin{aligned}
& \frac{1}{\tau \Gamma(2-\alpha)}\left[a_{0} v_{i}^{n}-\sum_{k=1}^{n-1}\left(a_{n-k-1}-a_{n-k}\right) v_{i}^{n}-a_{n-1} v_{i}^{0}\right] \\
& -\frac{1}{\tau \Gamma(2-\alpha)} \Delta\left[a_{0} v_{i}^{n}-\sum_{k=1}^{n-1}\left(a_{n-k-1}-a_{n-k}\right) v_{i}^{n}-a_{n-1} v_{i}^{0}\right] \\
& =\tau A_{i}^{n} \Delta\left(\sum_{k=1}^{n-1} v_{i}^{k}+\frac{v_{i}^{0}+v_{i}^{n}}{2}\right)-B_{i}^{n} \Delta \sigma_{i}^{n}, \\
& u_{i}^{n}(\Gamma)=\nabla u_{i}^{n}(\Gamma)=0, \quad 1 \leq i \leq m, \quad n \geq 1,
\end{aligned}
$$

have only zero solution. Using Lemma 4.1, we have

$$
\nabla u^{n}=0, \quad n=1, \cdots, N,
$$

and combining the above equality with the boundary condition in (2.4), we obtain

$$
u_{i}^{n}=v_{i}^{n}=0, \quad n \geq 1, \quad 1 \leq i \leq m .
$$

This completes the proof.

Theorem 4.2. Let $u(\mathbf{x}, t) \in C_{\mathbf{x}, t}^{4,3}(\Omega \times[0, T])$ and $\left\{v^{n} \mid n \geq 0\right\}$ be the solution of the difference scheme (2.2)-(2.3). Then, for $\tau<n \tau \leq T$, we have

$$
\left\|u\left(\mathbf{x}_{i}, t_{n}\right)-u_{i}^{n}\right\|_{\infty} \leq C^{*} D \sqrt{\Gamma(2-\alpha) T^{\alpha}}\left(\tau^{3-\alpha}+\hat{n}^{4-\min \{\hat{m}, p\}}\right),
$$

where $C^{*}$ is a constant number.

Proof. Denote

$$
\begin{aligned}
& \hat{v}_{i}^{n}=v\left(\mathbf{x}_{i}, t_{n}\right)-v_{i}^{n}, \\
& \hat{u}_{i}^{n}=u\left(\mathbf{x}_{i}, t_{n}\right)-u_{i}^{n}, \quad n \geq 0 .
\end{aligned}
$$

Subtracting (2.2-2.3) from (3.8) and (3.9) respectively, we have the error equations

$$
\begin{aligned}
& \frac{1}{\tau \Gamma(2-\alpha)}\left[a_{0} \hat{v}_{i}^{n}-\sum_{k=1}^{n-1}\left(a_{n-k-1}-a_{n-k}\right) \hat{v}_{i}^{n}-a_{n-1} \hat{v}_{i}^{0}\right] \\
& -\frac{1}{\tau \Gamma(2-\alpha)} \Delta\left[a_{0} \hat{v}_{i}^{n}-\sum_{k=1}^{n-1}\left(a_{n-k-1}-a_{n-k}\right) \hat{v}_{i}^{n}-a_{n-1} \hat{v}_{i}^{0}\right] \\
& =\tau A_{i}^{n} \Delta\left(\sum_{k=1}^{n-1} \hat{v}_{i}^{k}+\frac{\hat{v}_{0}+\hat{v}_{n}}{2}\right)-B_{i}^{n} \Delta \hat{\sigma}_{i}^{n}, \\
& \hat{u}_{i}^{n}(\Gamma)=\hat{v}_{i}^{n}(\Gamma)=\hat{\sigma}_{i}^{n}(\Gamma)=0, \quad n \geq 1 .
\end{aligned}
$$


Using Lemma 3.3, we have

$$
\left\|\nabla u^{n}\right\|^{2} \leq \frac{\Gamma(2-\alpha) t_{n}^{\alpha-1}}{4 \hat{C}} \tau \sum_{k=1}^{n}\left\|R^{k}\right\|^{2}, \quad \tau<n \tau \leq T .
$$

Inserting (3.8) into the right hand of the above inequality and considering the correction (2.10), we get

$$
\left|\nabla \hat{u}^{n}\right| \leq c^{*} \sqrt{\frac{D \Gamma(2-\alpha) T^{\alpha}}{4 \hat{C}}}\left(\tau^{3-\alpha}+\hat{n}^{4-\min \{\hat{m}, p\}}\right), \quad \tau<n \tau \leq T .
$$

Noticing (2.11), we have the result:

$$
\left\|\hat{u}^{n}\right\|_{\infty} \leq C^{*} D \sqrt{\Gamma(2-\alpha) T^{\alpha}}\left(\tau^{3-\alpha}+\hat{n}^{4-\min \{\hat{m}, p\}}\right), \quad \tau<n \tau \leq T,
$$

where $C^{*}$ are constant. This completes the proof.

Remark 4.1. Obviously, when $n$ is equal to 1 , the error order of the spatial variable $t$ is equal to $\mathcal{O}(\tau)$. But, by the increasing of $n$, the final error order will tend to $\mathcal{O}\left(\tau^{3-\alpha}\right)$ at $t=T$. The detailed conclusions are considered in the following numerical examples.

\section{Numerical experiments}

In this section, some comparative examples are provided to show the strength of the proposed method in approximating the solution of multi-dimensional time-fractional diffusion equations with a non-local boundary condition

In all numerical experiments, the presented method are local approximate schemes in spatial dimensions $\Omega$. The numerical results are performed in MATLAB 2014a on an Intel core i5(8G RAM) Windows Win10 system. The $L_{2}$ error which will be reported in those examples are defined as

$$
E_{n}=\max _{\mathbf{x}_{i} \in \Omega}\left|u^{n}\left(\mathbf{x}_{i}\right)-u_{i}^{n}\right| \text { and } \operatorname{Order}=\frac{\log _{2}\left(E_{n}\right)}{\log _{2}\left(E_{2 n}\right)},
$$

where $u_{i}$ and $u\left(\mathbf{x}_{i}\right)$ denote the numerical and exact solution of the problem respectively.

\subsection{D space fourth order fractional system}

Consider the following problem:

$$
\begin{aligned}
& { }_{0}^{c} \mathfrak{D}_{t}^{\alpha} u(x, t)+\left({ }_{0}^{c} \mathfrak{D}_{t}^{\alpha}-\cos (x t)\right) \Delta u(x, t)+\exp (x+t) \Delta^{2} u(x, t) \\
& =u^{3}(x, t)+f(x, t), \quad x \in(0,1), \quad t \in(0,1], \quad \alpha \in(1,2), \\
& u(0, t)=0 ; \quad u(1, t)=0, \quad \frac{\partial^{2} u(0, t)}{\partial x^{2}}=0, \quad \frac{\partial^{2} u(1, t)}{\partial x^{2}}=0, \\
& u(x, 0)=\sin (\pi x), \quad \frac{\partial u(x, 0)}{\partial t}=\sin (\pi x), \quad x \in(0,1), \quad t \in(0,1] .
\end{aligned}
$$



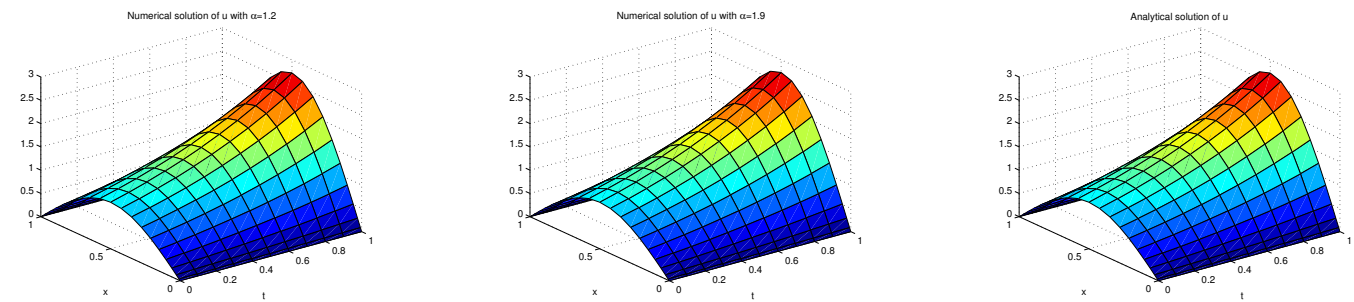

Figure 1: The surfaces for the numerical solutions (left and middle) and exact solutions (right).
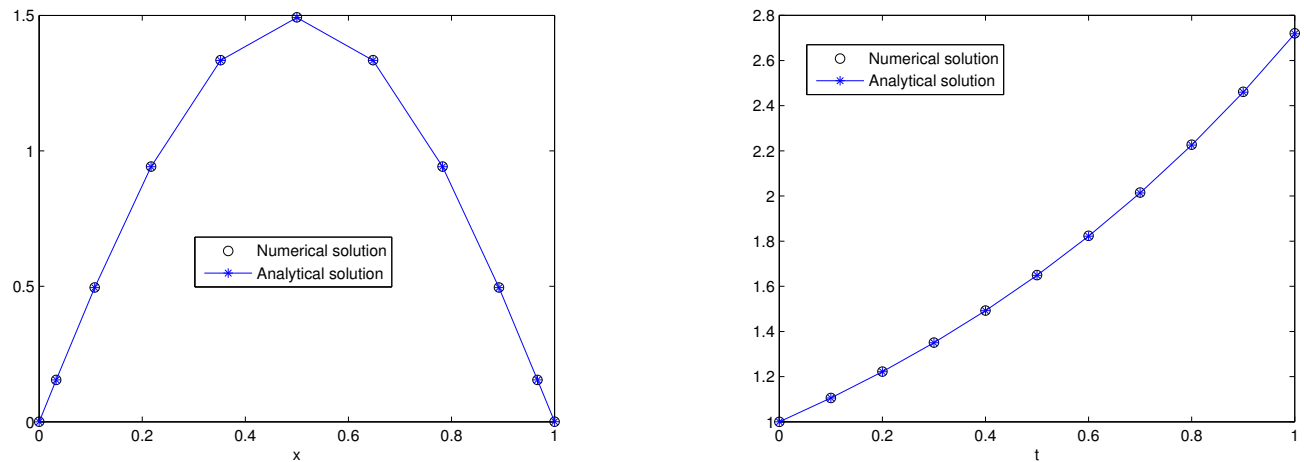

Figure 2: the curves of the numerical solutions and exact solutions along with $t=0.4$ (left) and $x=0.5$ (right).

The exact solution of the system is

$$
u(x, t)=\exp (t) \sin (\pi x)
$$

Take $\tau=1 / 10$ and $m=11$, Fig. 1 shows the numerical solutions (left and middle) of the proposed method and the exact solutions (right) at $t=1$ for the fourth order fractional diffusion-wave system for $\alpha=1.2$ (left) and $\alpha=1.9$ (middle) with $m=11$.

Fig. 2 plots the curves of the numerical solutions of the compact difference scheme and the analytical solutions of the same $\alpha,(\alpha=1.4)$ at the time $t=0.4$ and $x=0.5$. From these figures, it is easy to see that the proposed methods meets the analytical solution effectively.

Take $\alpha=1.7$, Table 1 represents some numerical and exact solutions on the points $(0.5,0.5),(0.5,1)$ for different mesh sizes with fixed temporal step. From there, we can see the presented scheme converges to the exact solutions quickly.

Table 2 give the temporal convergence order of the presented scheme at $t=1$ with $\alpha=1.9$, which are close to our theoretical values. 
Table 1: Some numerical results of the different grid schemes $(\tau=1 / 160)$.

\begin{tabular}{||c|ccc|ccc||}
\hline$(x, t)$ & $m=5$ & 7 & 9 & 11 & 13 & Exact solution \\
$(0.5,0.5)$ & 1.6530 & 1.6489 & 1.6489 & 1.6489 & 1.6489 & 1.6487 \\
$(0.5,1)$ & 2.7245 & 2.7184 & 2.7184 & 2.7184 & 2.7184 & 2.7183 \\
\hline
\end{tabular}

Table 2: Temporal convergence order of the presented scheme $(m=21)$.

\begin{tabular}{||cccccccc||}
\hline$\tau$ & $1 / 10$ & $1 / 20$ & $1 / 40$ & $1 / 80$ & $1 / 160$ & $1 / 320$ & Theoretical value \\
\hline$E_{n}$ & $1.0316 \mathrm{e}-2$ & $3.6200 \mathrm{e}-3$ & $1.3246 \mathrm{e}-3$ & $5.1886 \mathrm{e}-4$ & $2.1650 \mathrm{e}-4$ & $9.4577 \mathrm{e}-5$ & \\
Order & & 1.5109 & 1.4504 & 1.3521 & 1.2610 & 1.1948 & 1.1000 \\
\hline
\end{tabular}

\subsection{D space fourth order fractional system}

To implement the numerical effectiveness, we consider the following two dimensional nonlinear fourth-order equation

$$
\begin{aligned}
& { }_{0}^{c} \mathfrak{D}_{t}^{\alpha} u(x, y, t)+\left({ }_{0}^{c} \mathfrak{D}_{t}^{\alpha}-\cos (x y)\right) \Delta u(x, y, t)+\left(t^{2}+1\right) \Delta^{2} u(x, y, t) \\
& =u^{2}(x, y, t)-u(x, y, t)+f(x, y, t), \\
& \alpha \in(1,2), \quad(x, y) \in(0,1)^{2}, \quad t \in(0,1],
\end{aligned}
$$

with initial conditions and boundary conditions

$$
u(\Omega, 0)=0 ; \quad u_{t}(\Omega, 0)=\sin (2 \pi x) \sin (2 \pi y) ; \quad u(\Gamma, t)=0 ; \quad \sigma(\Gamma, t)=0, \quad t \in(0,1] .
$$

The exact solution of the system is

$$
u(x, y, t)=\left(t^{3+\alpha}+t\right) \sin (2 \pi x) \sin (2 \pi y) .
$$

Take $m=21^{2}, \alpha=1.3,1.5,1.7$, respectively. Table 3 gives some numerical results of the maximum errors and the corresponding temporal convergence order of the different temporal mesh size at $\mathrm{t}=1$, from which we can see the temporal convergence order is nearly and close to $\mathcal{O}\left(\tau^{3-\alpha}\right)$.

In Fig. 3, the surfaces of the numerical solution and the absolute error of $u$ are shown with $m=21^{2}, \tau=1 / 10$ and $\alpha=1.4$ at $t=1$. It not hard to see that the numerical solutions is vary close to the analytical solutions. Compared with the two surfaces of $u$, the surface of the absolute error coincides with the surface of numerical results. The similar conclusion between the numerical solution and absolute error of the $\sigma$ is obtained in Fig. 3.

\subsection{D space fourth order fractional system}

To implement the numerical effectiveness, we consider the following three dimensional nonlinear fourth-order equation

$$
\begin{aligned}
& { }_{0}^{c} \mathfrak{D}_{t}^{\alpha} u(x, y, z, t)+\left({ }_{0}^{c} \mathfrak{D}_{t}^{\alpha}-1\right) \Delta u(x, y, z, t)+\Delta^{2} u(x, y, z, t) \\
= & u^{3}(x, y, z, t)-u(x, y, z, t)+f(x, y, z, t), \quad \alpha \in(1,2), \quad(x, y, z) \in(0,1)^{3}, \quad t \in(0,1],
\end{aligned}
$$



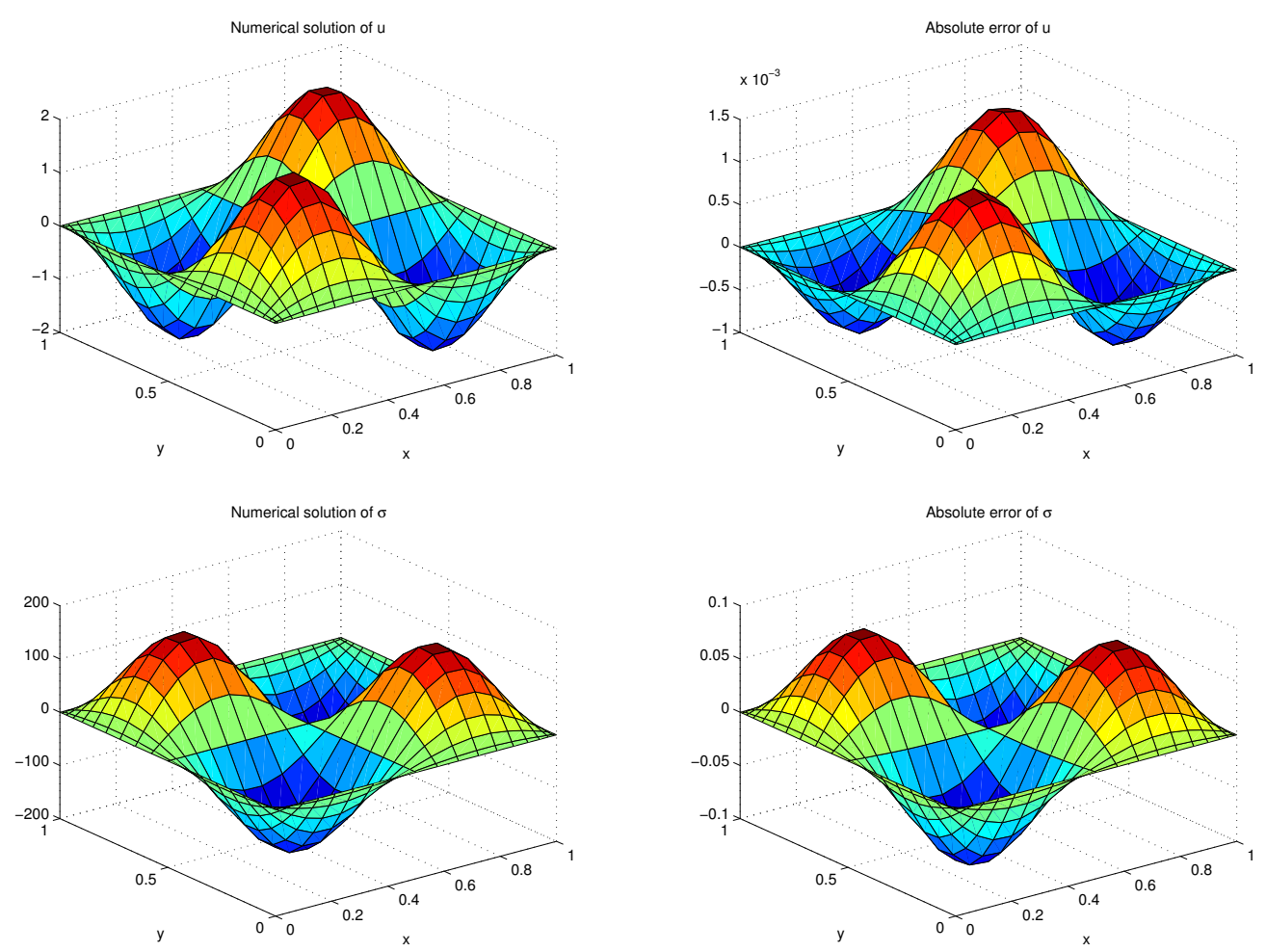

Figure 3: The surfaces of the numerical solution (left) and absolute error (right) of $u$ (up) and $\sigma$ (down).

Table 3: Some numerical results of the maximum errors and the corresponding temporal order at $t=1$ for the fourth order fractional equation.

\begin{tabular}{||c|cc|cc|cc||}
\hline \multirow{2}{*}{$\tau$} & \multicolumn{2}{|c|}{$\alpha=1.7$} & \multicolumn{2}{c|}{$\alpha=1.5$} & \multicolumn{2}{c||}{$\alpha=1.3$} \\
\cline { 2 - 7 } & $E_{n}$ & Order & $E_{n}$ & Order & $E_{n}$ & Order \\
\hline $1 / 10$ & $3.3835 \mathrm{e}-2$ & & $1.4740 \mathrm{e}-2$ & & $5.7372 \mathrm{e}-3$ & \\
$1 / 20$ & $1.5782 \mathrm{e}-2$ & 1.1002 & $5.2540 \mathrm{e}-3$ & 1.4882 & $1.7887 \mathrm{e}-3$ & 1.6814 \\
$1 / 40$ & $6.4484 \mathrm{e}-3$ & 1.2913 & $1.8611 \mathrm{e}-3$ & 1.4973 & $5.4500 \mathrm{e}-4$ & 1.7164 \\
$1 / 80$ & $2.6267 \mathrm{e}-3$ & 1.2957 & $6.8512 \mathrm{e}-4$ & 1.4997 & $1.6497 \mathrm{e}-4$ & 1.7241 \\
$1 / 160$ & $1.0685 \mathrm{e}-3$ & 1.2977 & $2.3267 \mathrm{e}-4$ & 1.5001 & $4.9872 \mathrm{e}-5$ & 1.7259 \\
$1 / 320$ & $4.3429 \mathrm{e}-4$ & 1.2989 & $8.2263 \mathrm{e}-5$ & 1.5000 & $1.5149 \mathrm{e}-5$ & 1.7190 \\
$1 / 640$ & $1.7645 \mathrm{e}-4$ & 1.2994 & $2.9087 \mathrm{e}-5$ & 1.4999 & $4.6189 \mathrm{e}-6$ & 1.7136 \\
$1 / 1280$ & $7.1680 \mathrm{e}-5$ & 1.2996 & $1.0285 \mathrm{e}-5$ & 1.4998 & $1.4126 \mathrm{e}-6$ & 1.7092 \\
$1 / 2560$ & $2.9114 \mathrm{e}-5$ & 1.2999 & $3.6367 \mathrm{e}-6$ & 1.4998 & $4.3292 \mathrm{e}-7$ & 1.7062 \\
\hline Theoretical value & & 1.3000 & & 1.5000 & & 1.7000 \\
\hline
\end{tabular}

with initial conditions and boundary conditions

$$
u(\Omega, 0)=0 ; \quad u_{t}(\Omega, 0)=0 ; \quad u(\Gamma, t)=0 ; \quad \sigma(\Gamma, t)=0, \quad t \in(0,1] .
$$



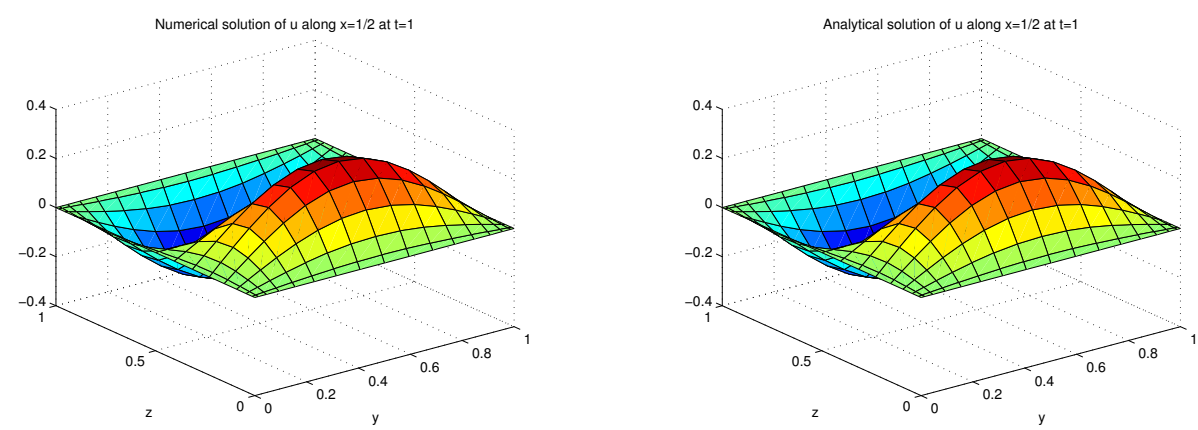

Figure 4: The surfaces for the numerical solutions (left) and exact solutions (right).

Table 4: Some numerical results of the maximum errors and the corresponding temporal order at $t=1$ for the fourth order fractional equation.

\begin{tabular}{||c|cc|cc|cc||}
\hline \multirow{2}{*}{$\tau$} & \multicolumn{2}{|c|}{$\alpha=1.3$} & \multicolumn{2}{c|}{$\alpha=1.5$} & \multicolumn{2}{c||}{$\alpha=1.7$} \\
\cline { 2 - 7 } & $E_{n}$ & Order & $E_{n}$ & Order & $E_{n}$ & Order \\
\hline $1 / 10$ & $7.7200 \mathrm{e}-4$ & & $1.8211 \mathrm{e}-3$ & & $4.1648 \mathrm{e}-3$ & \\
$1 / 20$ & $2.3963 \mathrm{e}-4$ & 1.6878 & $6.4596 \mathrm{e}-4$ & 1.4953 & $1.7144 \mathrm{e}-3$ & 1.2805 \\
$1 / 40$ & $7.4216 \mathrm{e}-5$ & 1.6910 & $2.2917 \mathrm{e}-4$ & 1.4950 & $6.9422 \mathrm{e}-4$ & 1.3042 \\
$1 / 80$ & $2.2945 \mathrm{e}-5$ & 1.6936 & $8.1211 \mathrm{e}-5$ & 1.4967 & $2.8068 \mathrm{e}-4$ & 1.3065 \\
$1 / 160$ & $7.0933 \mathrm{e}-6$ & 1.6937 & $2.8757 \mathrm{e}-5$ & 1.4978 & $1.1369 \mathrm{e}-4$ & 1.3038 \\
$1 / 320$ & $2.2197 \mathrm{e}-6$ & 1.6961 & $1.0182 \mathrm{e}-5$ & 1.4979 & $4.6115 \mathrm{e}-5$ & 1.3018 \\
\hline
\end{tabular}

The exact solution of the system is

$$
u(x, y, z, t)=\frac{t^{3}}{3} \sin (\pi x) \sin (\pi y) \sin (2 \pi z) .
$$

For the convenience of the numerical calculation, we now take $M=17^{3}$ and $\tau=1 / 20$.

In Fig. 4, Three comparisons for the surfaces of the exact solution and the numerical solution are shown along with $x=1 / 2$ at $t=1$ considering $\alpha=1.6$.

Take $\alpha=1.3,1.5,1.7$, respectively. Table 4 gives some numerical results of the maximum errors and the corresponding temporal convergence order of the different temporal mesh size at $\mathrm{t}=1$, from which we also take the conclusion that the temporal convergence order is nearly and close to $\mathcal{O}\left(\tau^{3-\alpha}\right)$.

\section{Conclusions}

In this paper, based on the Picard integral formulation, we present a compact finite difference scheme coupled with Chebyshev spectral collocation method to solve the multidimensional nonlinear variable coefficient time-fractional fourth-order partial differential 
system. Different from other many schemes, the proposed method consider the regularity of the derivative function $v$ on the integer time step. The stability and solvability are proved with detailed proofs and the precisely describe of error estimates is derived. To confirm the practicability and accuracy of the proposed method. several numerical results are calculated and analyzed in one-dimensional, two-dimensional and even three dimensional spatial domain. Numerical convergence rate consistent with the theoretical value $3-\alpha$ in $L_{\infty}$ norm.

\section{References}

[1] I. Podlubny, Fractional Differential Equations, Academic Press, New York, 1999.

[2] A. A. Kilbas, H. M. SRivastava and J. J. TRujillo, Theory and Applications of Fractional Differential Equations, North-Holland, New York, 2006.

[3] D. Baleanu, K. Diethelm, E. Scalas and J. J. Trujillo, Fractional Calculus Models and Numerical Methods (Series on Complexity, Nonlinearity and Chaos), World Scientific, 2012.

[4] A. A. Kilbas, H. M. SRivastava And J. J. Trujillo, Theory and Application of Fractional Differential Equations, Amsterdam, Elsevier, 2006.

[5] K. B. Oldham And J. Spanier, The Fractional Calculus, NewYork, London, Academic Press, 1974.

[6] X. LI AND C. XU, A space-time spectral method for the time fractional diffusion equation, SIAM J. Numer. Anal., 47 (2009), pp. 2108-2131.

[7] J. ZHAO, J. XIAO AND Y. XU, Stability and convergence of an effective finite element method for multiterm fractional partial differential equations, Abstr. Appl. Anal., (2013), pp. 1-10.

[8] P. Zhuang, Y. T. Gu, F. LiU, I. Turner And P. K. D. V. Yarlagadda, Time-dependent fractional advection-diffusion equations by an implicit MLS meshless method, Int. J. Numer. Methods Eng., 88 (2011), pp. 1346-1362.

[9] C. LI, Z. ZHAO AND Y. CHEN, Numerical approximation of nonlinear fractional differential equations with subdiffusion and super diffusion, Comput. Math. Appl., 62 (2011), pp. 855-875.

[10] F. LIU, P. ZHUANG, V. ANH AND I. TURNER, A fractional-order implicit difference approximation for the space time fractional diffusion equation, ANZIAMJ., 47 (2006), pp. 48-68.

[11] Z. ZHAO AND C. LI, Fractional differencelfinite element approximation for the time-space fractional telegraph equation, J. Appl. Math. Comput., 219 (2012), pp. 2975-2988.

[12] W. CHEN, H. SUN, X. ZHANG AND D. KOROŠAKB, Anomalous diffusion modeling by fractal and fractional derivatives, Comput. Math. Appl., 59 (2010), pp. 1754-1758.

[13] H. SUN, Y. ZHANG, W. CHEN AND D. M. REEVES, Use of a variable-index fractional-derivative model to capture transient dispersion in heterogeneous media, J. Contam. Hydrol., 157 (2014), pp. $47-58$.

[14] H. Sun, Z. P. LI, Y. ZHANG AND W. CHEN, Fractional and fractal derivative models for transient anomalous diffusion: Model comparison, Chaos Solitons Fractals, 102 (2017), pp. 346-353.

[15] A. GOLBABAI AND K. SAYEVAND, Fractional calculus-a new approach to the analysis of generalized fourth-order diffusion-wave equations, Comput. Math. Appl., 67 (2011), pp. 2227-2231.

[16] H. JAFARI, M. DEHGHAN AND K. SAYEVAND, Solving a fourth-order fractional diffusion wave equation in a bounded domain by decomposition method, Numer. Methods Partial Differ. Equ., 24 (2008), pp. 1115-1126. 
[17] O. P. AGRAWAL, A general solution for a fourth-order fractional diffusion-wave equation defined in a bounded domain, Comput. Struct., 79 (2001), pp. 1497-1501.

[18] S. VONG AND Z. WANG, Compact finite difference scheme for the fourth-order fractional subdiffusion system, Adv. Appl. Math. Mech., 6(4) (2014), pp. 419-435.

[19] X. L. HU AND L. M. ZHANG, On finite difference methods for fourth-order fractional diffusionwave and subdiffusion systems, Appl. Math. Comput., 218 (2012), pp. 5019-5034.

[20] Y. LiU, Y. W. DU, H. LI, J. C. LI AND S. HE, A two-grid mixed finite element method for a nonlinear fourth-order reaction diffusion problem with time-fractional derivative, Comput. Math. Appl., 70(10) (2015), pp. 2474-2492.

[21] Y. LIU, Y. W. DU, H. LI, S. HE AND W. GAO, Finite difference/finite element method for a nonlinear time-fractional fourth-order reaction-diffusion problem, Comput. Math. Appl., 70 (2015), pp. 573-591.

[22] J. LiN, W. CHEN AND F. Z. WANG, A new investigation into regularization techniques for the method of fundamental solutions, Math. Comput. Simulation, 18 (2011), pp. 1144-1152.

[23] J. Lin, C. S. CHEN, C. S. LIU AND J. LU, Fast simulation of multi-dimensional wave problems by the sparse scheme of the method of fundamental solutions, Comput. Math. Appl., 72(3) (2016), pp. 555-567.

[24] M. Dehghan, J. Manafian AND A. SAADATMAndi, The solution of the linear fractional partial differential equations using the homotopy analysis method, Zeitschriftfür NaturforschungA, 65(11) (2010), pp. 935-949.

[25] M. Dehghan, J. MANAFIAN AND A. SAADATMANDI, Solving nonlinear fractional partial differential equations using the homotopy analysis method, Numer. Methods Partial Differ. Equ., 26 (2010), pp. 448-479.

[26] F. LiU, M. M. Meerschaert, R. J. McGough, P. Zhuang AND Q. LiU, Numerical methods for solving the multi-term time-fractional wave-diffusion equation, Fract. Calc. Appl. Anal., 16 (2013), pp. 9-25.

[27] H. Zhang, F. Liu, M. S. Phanikumar and M. M. Meerschaert, A novel numerical method for the time variable fractional order mobile-immobile advectionCdispersion model, Comput. Math. Appl., 66 (2013), pp. 693-701.

[28] M. Parvizi, M. R. Eslahchi AND M. Dehghan, Numerical solution of fractional advectiondiffusion equation with a nonlinear source term, Numer. Algorithms, 68 (2015), pp. 601-629.

[29] J. P. BERRUT, Rational functions for guaranteed and experimentally well conditioned global interpolation, Comput. Math. Appl., 15(1) (1988), pp. 1-16.

[30] J. P. BERRUT, Baryzentrische formeln zur trigonometrischen interpolation I, Z. Angew. Math. Phys., 35 (1984), pp. 91-105.

[31] J. C. MASON, Chebyshev polynomials of the second, third and fourth kinds in approximation, indefinite integration and integral transforms, J. Comput. Appl. Math., 49 (1993), pp. 169-178.

[32] D. C. HANDSCOMB AND J. C. MASON, Chebyshev Polynomials, Chapman and Hall/CRC, BocaRaton, FL, 2003.

[33] G. KLEIN AND J. P. BERRUT, Linear rational finite differences from derivatives of barycentric rational interpolations, J. Numer. Anal., 50 (2012), pp. 643-656.

[34] R. BALTENSPERger, J. P. BERRUT AND B. NOEL, Exponential convergence of a linear rational interpolation between transformed Chebyshev points, Math. Comput., 227(28) (1999), pp. 11091120.

[35] J. P. BERRUT AND G. KLEIN, Recent advances in linear barycentric rational interpolation, J. Comput. Appl. Math., 259 (2014), pp. 95-107.

[36] O. R. ISIK, M. SEZER AND Z. GUNEY, A rational approximation based on Bernstein polynomials 
for high order initial and boundary values problems, Appl. Math. Comput., 217 (2011), pp. 94389450.

[37] T. HAut, G. Beylkin AND L. MONZON, Solving Burges' equation using optimal rational approximations, Appl. Comput. Harmon. Anal., 34 (2013), pp. 83-95.

[38] T. TANG AND J. JiE, Spectral and High-Order Methods with Applications, Science Press, 2006.

[39] Z. Z. SUN AND X. N. WU, A fully discrete difference scheme for a diffusion-wave system, Appl. Numer. Math., 56 (2006), pp. 193-209. 